\title{
Cytokines: How important are they in mediating
}

\section{sickness?}

\author{
David Chun-Hei POON ${ }^{1}$, Yuen-Shan $\mathrm{HO}^{1}$, Kin $\mathrm{CHIU}^{1}$, and \\ Raymond Chuen-Chung CHANG ${ }^{1,2,3 *}$ \\ ${ }^{1}$ Laboratory of Neurodegenerative Diseases, Department of Anatomy, \\ ${ }^{2}$ Research Centre of Heart, Brain, Hormone and Healthy Aging, \\ LKS Faculty of Medicine, \\ ${ }^{3}$ State Key Laboratory of Brain and Cognitive Sciences, \\ The University of Hong Kong, Pokfulam, Hong Kong SAR, CHINA
}

\author{
*Correspondence address: \\ Dr. Raymond C. C. Chang, Rm. L1-49, Laboratory Block, Faculty of Medicine \\ Building, 21 Sassoon Road, Pokfulam, Hong Kong SAR, CHINA \\ Tel: (+852) 2819-9127; Fax: (+852) 2817-0857; E-mail: rccchang@hku.hk
}


Sickness refers to a set of coordinated physiological and behavioral changes in response to systemic inflammation. It is characterized by fever, malaise, social withdrawal, fatigue, and annorexia. While these responses collectively represent a protective mechanism against infection and injury, increasing lines of evidence indicate that over-exaggerated or persistent sickness can damage the brain, and could possibly raise the risk to developing delirium. Therefore, a clear understanding in sickness will be beneficial. It has long been believed that sickness results from increased systemic cytokines occurring during systemic inflammation. However, in recent years more and more conflicting data have suggested that development of sickness following peripheral immune challenge could be independent of cytokines. Hence, it is confusing as to whether cytokines really do act as primary mediators of sickness, or if they are secondary to alternative inducing factor(s). In this review, we will (1) introduce the relationships between systemic inflammation, cytokines, sickness, and delirum, and (2) attempt to interpret the recent controversies.

Keywords: Cytokines; Sickness; Systemic inflammation; Delirium 


\section{Introduction}

We all prefer to live healthily and to be free of sickness. Getting sick because of infection (Campisi et al., 2003; Dantzer et al., 2008; Hart, 1988) or injury (Liu et al., 2008; Swain and Le, 1998) is discomforting and interferes greatly on our daily lives. While sickness was traditionally thought as a malfunction due to systemic inflammatory events, it is in fact an adaptive mechanism to facilitate recovery. This is mediated by a series highly coordinated physiological and behavioral changes, including fever, pain, fatigue, cognitive loss, anorexia, anhedonia, and social withdrawal (Dantzer, 2009; Dantzer et al., 2008; Hart, 1988), which will be referred to as sickness or sickness responses throughout this review. There is now ample evidence to show that these alterations can indeed help to fight infection (Hart, 1988). For example, an elevation in body temperature during fever is not only unfavorable to the growth of some pathogens, but also stimulates the activation and proliferation of immune cells. A reduction of appetite leads to a lowered intake of iron, which is important for the growth and replication of many pathogens. Nevertheless, while these responses are useful if well controlled, over-exaggerated sickness can be damaging. For instance, a persistent increase of brain temperature during fever might enhance neuronal excitotoxicity (Suehiro et al., 1999) and lead to abnormalities of 
blood-brain-barrier permeability (Sharma and Hoopes, 2003). A lowered food intake and greater thermogenesis would cause weight loss in a long run. Moreover, there is now evidence to suggest that dysregulated sickness responses, together with other risk factors such as aging and pre-exisiting dementia, could lead to delirium (de Rooij et 2007; Holmes et al., 2011). Hence, it will be advantageous if we can have a detailed understanding on the mechanisms by which sickness develops, and to implement suitable approaches to maximize their benefits without causing severe side effects.

Given that individual symptoms of sickness are regulated by specific regions in the brain, and that they are natural responses to systemic inflammation, it is to think that there are some biological signals relating the inflammatory events occurring at the periphery to the brain changes required for sickness responses. Over the last two decades, cytokines have emerged to be these linking signals. Accordingly, systemic inflammation triggers drastic releases of pro-inflammatory cytokines, including IL-1 $\beta$, IL-6, and TNF- $\alpha$, all of which can act on the brain, despite the blood-brain barrier, and lead to sickness. A number of neural and humoural routes are involved. Nevertheless, an increasing number of reports have shown that under some occasions, sickness following peripheral immune challenge could be independent of central and peripheral cytokine increases (Campisi et al., 2003; Murray et al., 2011; Teeling et al., 2010; Teeling et al., 2007). Therefore, we question the importance of 
cytokines, arising both systemically and within the brain, as mediators of sickness. the induction of cytokines alone be sufficient to cause sickness? Or are they simply secondary to alternative triggers of sickness?

Here, we summarize the relationships between systemic inflammation, cytokines, and sickness. Based on this background, we try to interpret the emerging controversial issues, and point out the gaps of knowledge. Moreover, since delirium may result over-exaggerated sickness (de Rooij et al., 2007; Holmes et al., 2011), we will also try to suggest possible roles of cytokines in delirum.

2. Cytokines are induced centrally and peripherally during systemic inflammation

Inflammation describes a cascade of vascular changes (e.g. vasodilation and increased capillary permeability) and cellular changes (e.g. recruitment and activation of immune cells) in response to infection and tissue injury. These physiological responses are orchestrated by increased levels of cytokines produced mainly by activated immune cells at the site of inflammation. Once produced, cytokines act both locally in autocrine and paracrine manners, and systemically at distant organs 2009; Kelso, 1998). For example, IFN- $\gamma$ stimulates tissue macrophages to up-regulate 
inducible nitric oxide synthase (iNOS) expression, leading to nitric oxide release (Blanchette et al., 2003). Nitric oxide in turn exerts antimicrobial (Mehta et al., 2012) and vasodilatory effects (Engelberger et al., 2011) in the microenvironment. Systemically, pro-inflammatory cytokines such as IL-1 $\beta$ and IL-6 produced at the inflammatory site are circulated to the liver, where they synergistically increase production of serum amyloid-A, which is involved in the complement cascade (Betts al., 1993). Yet, if we take a step further, we should ask an important question: How do immune cells sense invading pathogens and injured tissues and mount the cytokine responses for inflammation?

In respect to systemic infection, this is accomplished via the interactions between pathogen-associated molecular patterns (PAMPs) and pathogen recognition receptors (PRRs) (Bianchi, 2007; Kawai and Akira, 2009; Lee and Kim, 2007). PAMPs are conserved molecular motifs present in pathogens but are absent in the host, and can be distinguished as non-self by the immune system. PRRs are membrane bound or secretory receptors specific for these PAMPs, and they are expressed by many cell types such as monocyte/macrophage, natural killer cells, neutrophils, and cells. Binding of a PAMP to the corresponding PRR activates classical NF- $\kappa \mathrm{B}$, and IRF3 signaling pathways, leading to increased expression of pro-inflammatory cytokines such as IL-1 $\beta$, TNF- $\alpha$, IL-6, and interferons (Kawai and Akira, 2009). 
several classes of PRRs have been identified, including the Toll-like receptors (TLRs)

(Hedayat et al., 2011; Kawai and Akira, 2008), the nucleotide-binding oligomerization domain (NOD)-like receptors (NLRs) (Saleh, 2011), the retinoic acid-inducible gene-I (RIG-I)-like receptors (RLRs) (Kawai and Akira, 2008; Liu and Gu, 2011), and the C-type lectin receptors (CLRs) (Osorio and Reis e Sousa, 2011), and they are summarized in Table 1. For instance, lipopolysaccharide (LPS), which is a component of the outer membrane of gram negative bacteria and a commonly used immunostimulant in experimental setting, is a ligand for TLR4 and signals via a dependent manner (Medvedev et al., 2007).

Although tissue injury is often associated with a pathogenic stimulus, i.e. when one cuts his finger and exposes the underlying tissues to the limitless bacteria and viruses in the environment, injury alone can also trigger inflammation, a process as "sterile inflammation" (Chen and Nunez, 2010). An illustration of this is that after a person puts his finger on a hot stove, swelling and redness develop at the site. The mechanisms by which injury induces inflammation are less clear. It is that necrotic cells release endogenous danger signals known as alarmins, which act on innate immune cells to activate similar receptors (e.g. TLRs) and signaling pathways (e.g. NF- $\square$ B pathway) to those used by PPRs/PAMPs, leading to cytokine production (Bianchi, 2007; Rosin and Okusa, 2011). Some examples of alarmins and their 
receptors are listed in Table 2. Interestingly, IL-1 $\alpha$, a cytokine present normally as cytosolic or membrane bound forms, was found to be released from necrotic cell extracts, causing neutrophil recruitment in vivo and isolated mesothelial cells to produce CXCL1 and IL-6 (Eigenbrod et al., 2008).

Systemic inflammation not only induces cytokines systemically, but also within the brain. A good example is that systemic LPS challenge causes the brain (Quan et al., 1998; Singh and Jiang, 2004) and macrophage-like cells (Buttini et al., 1996; Eriksson et al., 2000; van Dam et al., 1992) to produce IL-1, which can diffuse the brain parenchyma (Vitkovic et al., 2000). Therefore, the brain can respond to PAMPs and alter its own cytokine profile.

In addition to the generation of cytokines to trigger inflammation, infections and injuries can induce sickness. This leads us to the next step: Do cytokines also regulate the development of sickness?

\section{The discovery of cytokines as mediators of sickness}

Following the development of recombinant cytokines in the 1980s, it was observed that patients and animals given an exogenous source of cytokines often developed flu-like symptoms and neuropsychiatric complications. For example, in a 
group of gastrointestinal cancer patients, intravenous injection of recombinant IL-1 $\beta$

resulted in fever, headache, rigors, nausea, and vomiting (Crown et al., 1991).

Moreover, around $20 \%$ of patients receiving long term IFN- $\alpha$ therapy for viral displayed neuropsychiatric symptoms like depression and altered consciousness (Renault et al., 1987). In animals, administration of IL-1 $\beta$ and TNF- $\alpha$ lead to a wide range of sickness responses, including fever, suppressed feeding, social withdrawal, immobility, and weight loss (Bluthe et al., 2000a; Dantzer et al., 2008; Kent et al., Palin et al., 2008; Stefferl et al., 1996). In contrast, injection of IL-6 alone into the induces a partial spectrum of sickness only, i.e. fever and reduced voluntary activity, but it could act synergistically with a sub-threshold dose of IL-1 $\beta$ to trigger anorexia and weight loss (Harden et al., 2008). Interestingly, similar sickness responses were observed when animals were given either central or systemic injections of bacterial LPS, which is an inducer of cytokines including IL-1 $\beta$, IL-6, and TNF- $\alpha$ in the brain and systemically (Bluthe et al., 1992; Henry et al., 2008; Huang et al., 2008). Taken together, these findings were suggestive for the idea that sickness induced by LPS resulted from increased systemic cytokines. However, this was not initially accepted because of the enormously high doses of cytokines used in the respective studies.

To confirm the effect of endogenous cytokines in mediating sickness, were done to verify whether deficiency of a single cytokine or its actions would blunt 
sickness responses to LPS. The involvement of endogenous IL-1 is quite clear, both systemic (Bluthe et al., 1992) and central (Laye et al., 2000) injections of IL-1 receptor antagonist (IL-1Ra) abrogated the suppressive effects of intraperitoneally injected LPS on social and/or feeding behaviors. Likewise, when compared to wild-type mice, IL-6 deficient mice exhibited less responsiveness to intraperitoneally and intracerebroventricularly injected LPS and IL-1 $\beta$ (Bluthe et al., 2000b), and in wild-type rats a pre-treatment with anti-IL-6 antibody abolished the effects of subcutaneously injected LPS on fever, voluntary running and food intake (Harden et 2006). More importantly, by blocking the actions of TNF- $\alpha$ within the brain via an intracerebroventricular injection of a fragment of the TNF soluble receptor, IL-1R1 deficient mice developed less severe sickness responses toward intraperitoneal injection of LPS (Bluthe et al., 2000a). This indicated that under physiological conditions, cytokines do not work independently, but rather functionally interact with each other in the form of cytokine networks to trigger different symptoms of sickness (Dantzer, 2009).

Thus, cytokines arising from inside and outside the brain modulate sickness. For cytokines arising systemically, they should signal back to the brain to take effects. However, since cytokines are hydrophilic molecules, they cannot freely pass through the blood-brain-barrier to enter the brain. This leads us to the next question: How do 
systemic cytokines affect the brain in the presence of a BBB?

\section{Systemic cytokines act on the brain via multiple routes}

There are many routes by which systemic cytokines act on the brain, and they be grossly classified into either being humoural dependent or neural dependent. Regarding the major humoural pathways, circulating cytokines can activate macrophage-like cells lining the circumventricular organs (CVO) (Dantzer, 2001, Dantzer et al., 2008; Schiltz and Sawchenko, 2002). These are brain regions that lack functional $\mathrm{BBB}$, including the median eminence (ME), organum vasculosum of the laminae terminalis (OVLT), area postrema (AP), and the suprafornical organ (SFO). Upon activation, these macrophage-like cells locally secrete high levels of cytokines, which can then enter the brain by volume diffusion (Vitkovic et al., 2000). increasing lines of evidence indicate that certain cytokines such as IL-1 (Banks et al., 2001; Banks et al., 1991), IL-1Ra (Gutierrez et al., 1994), TNF (Osburg et al., 2002), and IL-6 (Banks et al., 1994) can be selectively transported across the BBB in a blood-to-brain direction, mostly via saturable transport mechanisms. Circulating cytokines may also act on the brain vascular endothelium to trigger release of (An et al., 2011; Fabry et al., 1993; Thornton et al., 2010) and prostaglandin E2 
(Cao et al., 2001; Cao et al., 1996; Konsman et al., 2004). The latter binds to neuronal

$\mathrm{PGE}_{2}$ receptor $3(\mathrm{EP} 3)$ and $4(\mathrm{EP} 4)$ in the brainstem and hypothalamus, and regulates hypothalamic pituitary adrenal (HPA) axis activation and fever (Lazarus, 2006).

Evidence for the involvement of neural pathways in cytokine-mediated sickness was based on several major findings. To begin with, c-Fos is an immediate early gene and its expression has been commonly used as a marker of neuronal activation. An intraperitoneal injection of LPS, which potently stimulates cytokine production, leads to increased c-Fos immunopositive neurons at the primary (e.g. the nucleus tractus solitarius) and secondary projection areas (e.g. the supraoptic nucleus and paraventricular nucleus of the hypothalamus) of the vagus nerves (Dantzer, 2001; Dantzer et al., 2008). As the vagus nerve represents the major afferent pathway from the abdominal region to the brain, subdiaphragmatic vagotomy experiments were performed to see if this would reduce LPS-induced c-Fos expression. As expected, subdiaphragmatic vagotomy not only blocked LPS-induced brain c-Fos upregulation (Konsman et al., 2000; Wan et al., 1994), but also abolished LPS-induced brain IL-1 mRNA at the hippocampus and hypothalamus (Laye et al., 1995) and the decrease in social behavior (Bluthe et al., 1994; Konsman et al., 2000). Similarly, subdiaphragmatic vagotomy reduced social withdrawal and brain IL-1 $\beta$ mRNA expression following intraperitoneal IL-1 $\beta$ challenge (Hansen et al., 1998). Later, it 
was shown that an intraperitoneal LPS challenge up-regulated IL-1 $\beta$ immunoreactivity in immune cells associated with the abdominal vagus nerve (Goehler et al., 1999), vagus nerve sensory neurons express IL-1RI and intravenously injected IL-1 $\beta$ could stimulate vagus sensory activity (Ek et al., 1998), and that electrical stimulation of the vagus nerve elevated both brain IL-1 $\beta$ mRNA and protein levels and activated the HPA axis (Hosoi et al., 2000). Taken together, it would be reasonable to deduce that inflammation increases levels of IL-1 $\beta$, which then stimulates the vagus nerve to fire electrical signals back to the brain. These signals in turn up-regulate brain IL-1 $\beta$ expression, and could possibly modulate specific brain regions that control social behavior. However, subdiaphragmatic vagotomy did not reverse social withdrawal when IL-1 $\beta$ was injected via other administrative routes (Bluthe et al., 1996a, b). This indicated that the vagus nerve is likely to be only responsible for triggering sickness responses when inflammation takes place at the abdominal region but not at other peripheral sites.

The presence of both humoural dependent and neural dependent routes implies that the onset of sickness is regulated by several routes, and that different symptoms of sickness are also possibly controlled by separate routes. For example, subdiaphragmatic vagotomy prevented social withdrawal but not fever in response to LPS or IL-1 $\beta$ (Konsman et al., 2000). Also, it is obvious that cytokine messages can 
be conveyed more quickly to the brain through the neural dependent routes than through the humoural dependent routes. As suggested by Dantzer, systemic inflammation may first activate the fast neural routes, which sensitizes the brain to the subsequent actions from the slow humoural routes (Dantzer, 2001, 2009).

\section{Cytokine-induced sickness: Can we extend our perspectives to look at cytokines} in delirium?

Apart from mediating sickness, cytokines may also regulate delirium. Delirium, also commonly referred to as acute confusional state, is characterized by inattention, altered consciousness, cognitive deficits, hallucinations, and disorientation (Dasgupta and Hillier, 2010). It is often precipitated by systemic inflammation, and it occurs much more frequently in aged and dementia patients than in young and non-dementia individuals (Murray et al., 2012). More importantly, delirium is strongly associated with prolonged hospitalization time and costs, greater subsequent cognitive decline, and increased mortality (Fong et al., 2009).

There is evidence to suggest that delirium may result from over-exaggerated sickness, and because cytokines are sickness mediators they may also participate in delirium (de Rooij et al., 2007; Holmes et al., 2011). Firstly, cytokines are increased 
in delirious patients, and in particular blood IL-6 and IL-8 (de Rooij et al., 2007; van Munster et al., 2010; van Munster et al., 2008), CSF IL-8 (Hall et al., 2011), and brain IL-6 immunoreactivity (Munster et al., 2011) were found to be significantly higher in delirious patients than in non-delirious patients. Secondly, both sickness and delirium can be triggered by systemic inflammation, and they share several overlapping symptoms, including cognitive disturbances, decreased concentration, and apathy (Dasgupta and Hillier, 2010; Holmes et al., 2011). Thirdly, delirium can result from long-term IFN- $\alpha$ therapy in patients with chronic viral hepatitis (Renault et al., 1987), and that IL-1 suppresses cholinergic pathways (Li et al., 2000), which are also dysfunctional in delirium (Flacker and Lipsitz, 1999). Fourthly, while systemic inflammation leads to sickness in normal individuals, it frequently precipitates delirium in Alzheimer's disease patients (Holmes et al., 2011). This is thought to be because neurodegeneration in Alzheimer's disease causes "priming" of brain microglia, which would synthesize much more cytokines (e.g. IL-1 $\beta$ ) upon systemic inflammatory insults (Chang et al., 2009; Cunningham et al., 2009; Field et al., 2010; Perry, 2010).

Owing to the lack of well established animal models of delirium, the possible links between cytokines and delirium is in fact still at its infancy. Until recently, the ME7 prion diseased mice have been suggested to be a suitable animal model for 
delirium (Murray et al., 2012). At present stage, the urgent question we should ask is how we could extend our knowledge from cytokine-induced sickness to the relationships between cytokines and delirium, as summarized in Figure 1, and if this could provide insights on the prevention, treatment, and evaluation of recovery for delirium. For example, if cytokines are involved in delirium, then which cytokines can be biomarkers to predict whether a patient is likely to develop delirium, and which cytokines can be used to evaluate patients' recovery after delirium? There is already a report suggesting that peak levels of blood IL-8 and IL-6 occur before and during delirium respectively in elderly patients with hip fractures (van Munster et al., 2010; van Munster et al., 2008). Furthermore, low insulin-like growth factor 1 (IGF-1) and high initial IFN- $\gamma$ levels in blood are correlated with the incidence and recovery of delirium, respectively (Adamis et al., 2007). These studies are certainly in the correct direction, and we will need more cross-sectional analyses to test whether these cytokines are also associated with delirium under other inflammatory causes. On the other hand, it was found that dexamethasone could not reduce LPS-induced sickness despite blocking blood cytokine increases (Murray et al., 2011; Teeling et al., 2010), This suggests that targeting circulating cytokines levels may be ineffective against delirium, although data on manipulation of other key cytokines such as IL-8 still lacking. 
In the next section, we will highlight several interesting examples in which cytokines may appear to be disconnected to sickness development. Our purpose is not to against the model of cytokine-induced sickness, but rather we would like to stimulate our readers to not only redefine the importance of cytokines in mediating sickness, but also on top of that to explore the significance of cytokines in delirium.

\section{Cytokines: Can they explain everything in sickness?}

In previous sections, we have provided multiple lines of evidence to support the model of cytokine-induced sickness. Firstly, systemic inflammation, either because of infection or tissue injury, causes heightened levels of cytokines in the brain and at the periphery. Secondly, both exogenously administered and endogenously produced cytokines are involved in the induction and resolution of sickness responses, e.g. fever, social withdrawal, suppressed feeding. Thirdly, systemic cytokines communicate with the brain through a number of fast neural and slow humoural routes to trigger neuronal activation, increased expression of mRNA for brain cytokines, and production of $\mathrm{PGE}_{2}$. What remains mostly unknown is how cytokines bring about sickness responses at cellular and at molecular levels within the brain, but this would not rule out their roles in regulating sickness. The big question we should ask is how 
important cytokines are as mediators of sickness.

In order to answer this question, it would be easier if we first think about it from another perspective: can we get sick without the effects of cytokines? In fact, from the literature there are several reports suggesting that sickness may develop independently of cytokines (Campisi et al., 2003; Murray et al., 2011; Teeling et al., 2010; Teeling et al., 2007). These surprising findings have enlightened us to rethink about the current model of cytokine-induced sickness, and led us to share our opinions on the importance of cytokines in mediating sickness in this review.

While most studies have reported that the elevations of cytokines in blood and the brain occur before or at the same time as the onset of sickness responses, this is not mandatory in all cases. For example, in one study a subcutaneous injection of E.coli in rats caused fever and reduced activity starting from the $4^{\text {th }}$ hour, before the increases of cytokines in the brain and plasma beginning at the $6^{\text {th }}$ hour, and initial rise of circulating endotoxins at the $18^{\text {th }}$ hour (Campisi et al., 2003). Hence, both blood-borne cytokines and endotoxins were not involved in initiating sickness in this study. However, there was increased local cytokine synthesis at the injection site as early as $2^{\text {nd }}$ hour. Hence, prior to spilling over to the bloodstream and to be carried to the brain, these cytokines could have directly activated neighboring nerve terminals of afferent nerves, which immediately transmitted signals back to the brain to stimulate 
the neurons controlling fever and activity, before brain cytokines were up-regulated.

In support of this, the vagus (Bluthe et al., 1996a, b) and the glossopharygeal nerves (Romeo et al., 2001) are respectively responsible for communicating inflammatory signals from the abdominal and oral cavities to the brain. It would be expectable that other afferent nerve(s) would also be involved in communicating subcutaneous inflammatory signals back to the brain. Furthermore, even though blood-borne cytokines were not required for initiating sickness, activation of the fast neural routes may sensitize the brain to the effects of the slow humoural routes (Dantzer, 2001, 2009). Importantly, since fever and reduced activity persisted as long as brain and plasma cytokines were still elevated, cytokines were still likely to be required to sustain sickness responses during an infection. Therefore, it should be noted that the induction of circulating and brain cytokines do not necessarily have to occur before the onset of sickness responses, even though cytokines do mediate sickness.

Another striking observation is that sickness responses can be effectively reduced without suppressing central and/or peripheral cytokine levels. In studies performed by Teeling et al, pretreatment of mice with indomethacin, which is a non-selective COX inhibitor and inhibits prostaglandins production, abrogated the depressive effect of an intraperitoneal injection of a subpyrogenic dose of LPS on burrowing activity (Teeling et al., 2007). Likewise, in another study pretreatment of 
mice with a selective COX-1 inhibitor piroxicram, but not a selective COX-2 inhibitor nimesulide, attenuated the reductions of burrowing and open-field activity in response to a high dose of LPS injected intraperioneally (Teeling et al., 2010). Since in both studies administration of COX inhibitors were able to down-regulate LPS-mediated sickness behaviors without causing significant differences in plasma and brain cytokine responses, they highlight the pivotal roles of COX in controlling sickness. Nevertheless, we cannot conclude that cytokines are not important mediators of sickness. The induction of cytokines and COX-dependent products could act together at the brain to achieve a full spectrum of sickness responses (Blatteis et al., 2005; Pecchi et al., 2006).

The last intriguing observation is that blocking increase of plasma cytokines or their systemic actions is not necessarily sufficient to suppress all symptoms of sickness. For instance, pretreatment with dexamethasone-21-phosphate or dexamethasone prevented peripheral LPS-induced hypothermia and the elevations of circulating cytokines, but it could not inhibit the decrements in burrowing, rearing, and open field activity (Murray et al., 2011; Teeling et al., 2010). Similarly, systemic injection of neutralizing antibodies against IL-1 $\beta$, IL-6, or TNF- $\alpha$ before LPS treatment did not reverse the drop in burrowing or attenuate any increase of brain cytokine mRNA (Teeling et al., 2007), although in another study a pretreatment with 
anti-IL- 6 but not anti-IL-1 $\beta$ antisera suppressed LPS-induced fever, anorexia, and the reduction in voluntary wheel running (Harden et al., 2006). Hence, solely managing plasma cytokines would not be a good therapeutic strategy to reduce sickness, especially if only one cytokine is reduced because cytokines share many redundant effects.

Therefore, these studies are not only supportive for cytokine-induced sickness, but they also highlighted several key aspects of this model that are often neglected. Firstly, while most studies have measured cytokines in blood and at the brain, it is worth mentioning that cytokines arising at the inflammatory site could already signal to the brain via the fast neural routes and possibly be sufficient to initiate sickness (Campisi et al., 2003). This former event precedes the increases of plasma and brain cytokines, and probably also sensitizes the brain to the effects of the latter (Dantzer, 2001, 2009). Secondly, although cytokines do regulate sickness, they are not the only factor governing sickness development. Other factors such as $\mathrm{PGE}_{2}$ could alone or synergistically act with cytokines to elicit a full-blown sickness (Lazarus, 2006; Teeling et al., 2010; Teeling et al., 2007). Thirdly, drugs that primarily target at the plasma cytokine profile are not ideal to control sickness severity, as demonstrated in the example of dexamethasone or dexamethasone-21 treatment towards LPS-induced sickness (Murray et al., 2011; Teeling et al., 2010). 
The model of cytokine-induced sickness has been well established. Our next step should characterize how important cytokines are in mediating sickness under real life infections and injuries. Can pathological levels of cytokines alone be sufficient to initiate some, if not all, symptoms of sickness? To date, numerous animal experiments have studied the behavior and biochemical effects of either individual exogenous cytokines at doses that cannot be reached by the body, or high amounts of purified PAMPs, e.g. LPS, which elicit many non-cytokine effects in animals. Their findings are certainly essential to our current understanding of cytokine-induced sickness, but they are not necessarily translatable to real life situations. Given that in vivo cytokines exert their effects at picomolar to nanomolar concentrations, and that multiple cytokines are simultaneously up-regulated and functionally interact to form cytokine networks, we suggest that our research should not just focus at the effects of mega doses of one single cytokine at a time, but instead should move to administering lower and physiologically reachable doses of multiple cytokines to see their combined effects. Hopefully, this will also shed light on unraveling the importance of cytokines in delirium.

\section{Conclusion}



substantial knowledge in how systemic inflammation up-regulates cytokine levels, the effects of cytokines on behavior and fever, and the mechanisms by which systemic cytokines act at the brain. What we still need to clarify is how cytokines influence the brain at cellular and molecular levels, and the relative importance of cytokines, as compared to other mediators, in sickness during real life infections and injuries. Moreover, delirium can be regarded as an extremity of sickness. While systemic inflammation causes sickness in young and non-dementia individuals, it precipitates delirium in the aged and dementia patients. Whether elevated cytokines during systemic inflammation contribute significantly to delirium is still unclear, and this should also be our focus in the future. 


\title{
Acknowledgement
}

\author{
The work on systemic cytokines and the brain in this laboratory is supported by \\ Research Fund for the Control of Infectious Diseases (RFCID) of Food and Health \\ Bureau, Hong Kong SAR government (09090922), and University (HKU) \\ Alzheimer's Disease Research Network under Strategic Research Theme on Healthy \\ Aging.
}




\section{References}

Adamis, D., Treloar, A., Martin, F.C., Gregson, N., Hamilton, G., Macdonald, A.J., 2007. APOE and cytokines as biological markers for recovery of prevalent delirium in elderly medical inpatients. Int J Geriatr Psychiatry 22, 688-694.

An, Y., Chen, Q., Quan, N., 2011. Interleukin-1 exerts distinct actions on different cell types of the brain in vitro. J Inflamm Res 2011, 11-20.

Banks, W.A., Farr, S.A., La Scola, M.E., Morley, J.E., 2001. Intravenous human interleukin-1alpha impairs memory processing in mice: dependence on blood-brain barrier transport into posterior division of the septum. J Pharmacol Exp Ther 299, $536-541$.

Banks, W.A., Kastin, A.J., Gutierrez, E.G., 1994. Penetration of interleukin-6 across the murine blood-brain barrier. Neurosci Lett 179, 53-56.

Banks, W.A., Ortiz, L., Plotkin, S.R., Kastin, A.J., 1991. Human interleukin (IL) 1 alpha, murine IL-1 alpha and murine IL-1 beta are transported from blood to brain in the mouse by a shared saturable mechanism. J Pharmacol Exp Ther 259, 988-996.

Betts, J.C., Cheshire, J.K., Akira, S., Kishimoto, T., Woo, P., 1993. The role of NF-kappa B and NF-IL6 transactivating factors in the synergistic activation of human serum amyloid A gene expression by interleukin-1 and interleukin-6. J Biol Chem 268, 25624-25631. 
Bianchi, M.E., 2007. DAMPs, PAMPs and alarmins: all we need to know about danger. J Leukoc Biol 81, 1-5.

Blanchette, J., Jaramillo, M., Olivier, M., 2003. Signalling events involved in interferon-gamma-inducible macrophage nitric oxide generation. Immunology 108, $513-522$.

Blatteis, C.M., Li, S., Li, Z., Feleder, C., Perlik, V., 2005. Cytokines, PGE2 and endotoxic fever: a re-assessment. Prostaglandins Other Lipid Mediat 76, 1-18.

Bluthe, R.M., Dantzer, R., Kelley, K.W., 1992. Effects of interleukin-1 receptor antagonist on the behavioral effects of lipopolysaccharide in rat. Brain Res 573, 318-320.

Bluthe, R.M., Laye, S., Michaud, B., Combe, C., Dantzer, R., Parnet, P., 2000a. Role of interleukin-1beta and tumour necrosis factor-alpha in lipopolysaccharide-induced sickness behaviour: a study with interleukin-1 type I receptor-deficient mice. Eur J Neurosci 12, 4447-4456.

Bluthe, R.M., Michaud, B., Kelley, K.W., Dantzer, R., 1996a. Vagotomy attenuates behavioural effects of interleukin-1 injected peripherally but not centrally. Neuroreport 7, 1485-1488.

Bluthe, R.M., Michaud, B., Kelley, K.W., Dantzer, R., 1996b. Vagotomy blocks behavioural effects of interleukin-1 injected via the intraperitoneal route but not via 
other systemic routes. Neuroreport 7, 2823-2827.

Bluthe, R.M., Michaud, B., Poli, V., Dantzer, R., 2000b. Role of IL-6 in cytokine-induced sickness behavior: a study with IL-6 deficient mice. Physiol Behav $70,367-373$.

Bluthe, R.M., Walter, V., Parnet, P., Laye, S., Lestage, J., Verrier, D., Poole, S., Stenning, B.E., Kelley, K.W., Dantzer, R., 1994. Lipopolysaccharide induces sickness behaviour in rats by a vagal mediated mechanism. C R Acad Sci III 317, 499-503.

Buttini, M., Limonta, S., Boddeke, H.W., 1996. Peripheral administration of lipopolysaccharide induces activation of microglial cells in rat brain. Neurochem Int $29,25-35$.

Campisi, J., Hansen, M.K., O'Connor, K.A., Biedenkapp, J.C., Watkins, L.R., Maier, S.F., Fleshner, M., 2003. Circulating cytokines and endotoxin are not necessary for the activation of the sickness or corticosterone response produced by peripheral E. coli challenge. J Appl Physiol 95, 1873-1882.

Cao, C., Matsumura, K., Shirakawa, N., Maeda, M., Jikihara, I., Kobayashi, S., Watanabe, Y., 2001. Pyrogenic cytokines injected into the rat cerebral ventricle induce cyclooxygenase-2 in brain endothelial cells and also upregulate their receptors. Eur $\mathbf{J}$ Neurosci 13, 1781-1790.

Cao, C., Matsumura, K., Yamagata, K., Watanabe, Y., 1996. Endothelial cells of the 
rat brain vasculature express cyclooxygenase- 2 mRNA in response to systemic interleukin-1 beta: a possible site of prostaglandin synthesis responsible for fever. Brain Res 733, 263-272.

Chang, R.C., Chiu, K., Ho, Y.S., So, K.F., 2009. Modulation of neuroimmune responses on glia in the central nervous system: implication in therapeutic intervention against neuroinflammation. Cell Mol Immunol 6, 317-326.

Chen, G.Y., Nunez, G., 2010. Sterile inflammation: sensing and reacting to damage. Nat Rev Immunol 10, 826-837.

Crown, J., Jakubowski, A., Kemeny, N., Gordon, M., Gasparetto, C., Wong, G., Sheridan, C., Toner, G., Meisenberg, B., Botet, J., et al., 1991. A phase I trial of recombinant human interleukin-1 beta alone and in combination with myelosuppressive doses of 5-fluorouracil in patients with gastrointestinal cancer. Blood 78, 1420-1427.

Cunningham, C., Campion, S., Lunnon, K., Murray, C.L., Woods, J.F., Deacon, R.M., Rawlins, J.N., Perry, V.H., 2009. Systemic inflammation induces acute behavioral and cognitive changes and accelerates neurodegenerative disease. Biol Psychiatry 65, 304-312.

Dantzer, R., 2001. Cytokine-induced sickness behavior: where do we stand? Brain Behav Immun 15, 7-24. 
Dantzer, R., 2009. Cytokine, sickness behavior, and depression. Immunol Allergy Clin North Am 29, 247-264.

Dantzer, R., O'Connor, J.C., Freund, G.G., Johnson, R.W., Kelley, K.W., 2008. From inflammation to sickness and depression: when the immune system subjugates the brain. Nat Rev Neurosci 9, 46-56.

Dasgupta, M., Hillier, L.M., 2010. Factors associated with prolonged delirium: a systematic review. Int Psychogeriatr 22, 373-394.

de Rooij, S.E., van Munster, B.C., Korevaar, J.C., Levi, M., 2007. Cytokines and acute phase response in delirium. J Psychosom Res 62, 521-525.

Eigenbrod, T., Park, J.H., Harder, J., Iwakura, Y., Nunez, G., 2008. Cutting edge: critical role for mesothelial cells in necrosis-induced inflammation through the recognition of IL-1 alpha released from dying cells. J Immunol 181, 8194-8198.

Ek, M., Kurosawa, M., Lundeberg, T., Ericsson, A., 1998. Activation of vagal afferents after intravenous injection of interleukin-1beta: role of endogenous prostaglandins. J Neurosci 18, 9471-9479.

Engelberger, R.P., Pittet, Y.K., Henry, H., Delodder, F., Hayoz, D., Chiolero, R.L., Waeber, B., Liaudet, L., Berger, M.M., Feihl, F., 2011. Acute endotoxemia inhibits microvascular nitric oxide-dependent vasodilation in humans. Shock 35, 28-34.

Eriksson, C., Nobel, S., Winblad, B., Schultzberg, M., 2000. Expression of interleukin 
1 alpha and beta, and interleukin 1 receptor antagonist mRNA in the rat central nervous system after peripheral administration of lipopolysaccharides. Cytokine 12, 423-431.

Fabry, Z., Fitzsimmons, K.M., Herlein, J.A., Moninger, T.O., Dobbs, M.B., Hart, M.N., 1993. Production of the cytokines interleukin 1 and 6 by murine brain microvessel endothelium and smooth muscle pericytes. J Neuroimmunol 47, 23-34.

Field, R., Campion, S., Warren, C., Murray, C., Cunningham, C., 2010. Systemic challenge with the TLR3 agonist poly I:C induces amplified IFNalpha/beta and IL-1beta responses in the diseased brain and exacerbates chronic neurodegeneration. Brain Behav Immun 24, 996-1007.

Flacker, J.M., Lipsitz, L.A., 1999. Serum anticholinergic activity changes with acute illness in elderly medical patients. J Gerontol A Biol Sci Med Sci 54, M12-16.

Fong, T.G., Tulebaev, S.R., Inouye, S.K., 2009. Delirium in elderly adults: diagnosis, prevention and treatment. Nat Rev Neurol 5, 210-220.

Goehler, L.E., Gaykema, R.P., Nguyen, K.T., Lee, J.E., Tilders, F.J., Maier, S.F., Watkins, L.R., 1999. Interleukin-1beta in immune cells of the abdominal vagus nerve: a link between the immune and nervous systems? J Neurosci 19, 2799-2806.

Gutierrez, E.G., Banks, W.A., Kastin, A.J., 1994. Blood-borne interleukin-1 receptor antagonist crosses the blood-brain barrier. J Neuroimmunol 55, 153-160. 
Hall, R.J., Shenkin, S.D., Maclullich, A.M., 2011. A systematic literature review of cerebrospinal fluid biomarkers in delirium. Dement Geriatr Cogn Disord 32, 79-93.

Hansen, M.K., Taishi, P., Chen, Z., Krueger, J.M., 1998. Vagotomy blocks the induction of interleukin-1beta (IL-1beta) mRNA in the brain of rats in response to systemic IL-1beta. J Neurosci 18, 2247-2253.

Harden, L.M., du Plessis, I., Poole, S., Laburn, H.P., 2006. Interleukin-6 and leptin mediate lipopolysaccharide-induced fever and sickness behavior. Physiol Behav 89, 146-155.

Harden, L.M., du Plessis, I., Poole, S., Laburn, H.P., 2008. Interleukin (IL)-6 and IL-1 beta act synergistically within the brain to induce sickness behavior and fever in rats. Brain Behav Immun 22, 838-849.

Hart, B.L., 1988. Biological basis of the behavior of sick animals. Neurosci Biobehav Rev 12, 123-137.

Hedayat, M., Netea, M.G., Rezaei, N., 2011. Targeting of Toll-like receptors: a decade of progress in combating infectious diseases. Lancet Infect Dis 11, 702-712.

Henry, C.J., Huang, Y., Wynne, A., Hanke, M., Himler, J., Bailey, M.T., Sheridan, J.F., Godbout, J.P., 2008. Minocycline attenuates lipopolysaccharide (LPS)-induced neuroinflammation, sickness behavior, and anhedonia. J Neuroinflammation 5, 15.

Holmes, C., Cunningham, C., Zotova, E., Culliford, D., Perry, V.H., 2011. 
Proinflammatory cytokines, sickness behavior, and Alzheimer disease. Neurology 77, 212-218.

Hosoi, T., Okuma, Y., Nomura, Y., 2000. Electrical stimulation of afferent vagus nerve induces IL-1beta expression in the brain and activates HPA axis. Am J Physiol Regul Integr Comp Physiol 279, R141-147.

Huang, Y., Henry, C.J., Dantzer, R., Johnson, R.W., Godbout, J.P., 2008. Exaggerated sickness behavior and brain proinflammatory cytokine expression in aged mice in response to intracerebroventricular lipopolysaccharide. Neurobiol Aging 29, 1744-1753.

Kawai, T., Akira, S., 2008. Toll-like receptor and RIG-I-like receptor signaling. Ann N Y Acad Sci 1143, 1-20.

Kawai, T., Akira, S., 2009. The roles of TLRs, RLRs and NLRs in pathogen recognition. Int Immunol 21, 317-337.

Kelso, A., 1998. Cytokines: principles and prospects. Immunol Cell Biol 76, 300-317. Kent, S., Bluthe, R.M., Dantzer, R., Hardwick, A.J., Kelley, K.W., Rothwell, N.J., Vannice, J.L., 1992. Different receptor mechanisms mediate the pyrogenic and behavioral effects of interleukin 1. Proc Natl Acad Sci U S A 89, 9117-9120.

Konsman, J.P., Luheshi, G.N., Bluthe, R.M., Dantzer, R., 2000. The vagus nerve mediates behavioural depression, but not fever, in response to peripheral immune 
signals; a functional anatomical analysis. Eur J Neurosci 12, 4434-4446.

Konsman, J.P., Vigues, S., Mackerlova, L., Bristow, A., Blomqvist, A., 2004. Rat brain vascular distribution of interleukin-1 type-1 receptor immunoreactivity: relationship to patterns of inducible cyclooxygenase expression by peripheral inflammatory stimuli. J Comp Neurol 472, 113-129.

Laye, S., Bluthe, R.M., Kent, S., Combe, C., Medina, C., Parnet, P., Kelley, K., Dantzer, R., 1995. Subdiaphragmatic vagotomy blocks induction of IL-1 beta mRNA in mice brain in response to peripheral LPS. Am J Physiol 268, R1327-1331.

Laye, S., Gheusi, G., Cremona, S., Combe, C., Kelley, K., Dantzer, R., Parnet, P., 2000. Endogenous brain IL-1 mediates LPS-induced anorexia and hypothalamic cytokine expression. Am J Physiol Regul Integr Comp Physiol 279, R93-98.

Lazarus, M., 2006. The differential role of prostaglandin E2 receptors EP3 and EP4 in regulation of fever. Mol Nutr Food Res 50, 451-455.

Lee, M.S., Kim, Y.J., 2007. Signaling pathways downstream of pattern-recognition receptors and their cross talk. Annu Rev Biochem 76, 447-480.

Li, Y., Liu, L., Kang, J., Sheng, J.G., Barger, S.W., Mrak, R.E., Griffin, W.S., 2000. Neuronal-glial interactions mediated by interleukin-1 enhance neuronal acetylcholinesterase activity and mRNA expression. J Neurosci 20, 149-155.

Liu, F., Gu, J., 2011. Retinoic acid inducible gene-I, more than a virus sensor. Protein 
Cell 2, 351-357.

Liu, M., Liang, Y., Chigurupati, S., Lathia, J.D., Pletnikov, M., Sun, Z., Crow, M., Ross, C.A., Mattson, M.P., Rabb, H., 2008. Acute kidney injury leads to inflammation and functional changes in the brain. J Am Soc Nephrol 19, 1360-1370.

Medvedev, A.E., Piao, W., Shoenfelt, J., Rhee, S.H., Chen, H., Basu, S., Wahl, L.M., Fenton, M.J., Vogel, S.N., 2007. Role of TLR4 tyrosine phosphorylation in signal transduction and endotoxin tolerance. J Biol Chem 282, 16042-16053.

Mehta, D.R., Ashkar, A.A., Mossman, K.L., 2012. The nitric oxide pathway provides innate antiviral protection in conjunction with the type I interferon pathway in fibroblasts. PLoS One 7, e31688.

Munster, B.C., Aronica, E., Zwinderman, A.H., Eikelenboom, P., Cunningham, C., Rooij, S.E., 2011. Neuroinflammation in delirium: a postmortem case-control study. Rejuvenation Res 14, 615-622.

Murray, C., Sanderson, D.J., Barkus, C., Deacon, R.M., Rawlins, J.N., Bannerman, D.M., Cunningham, C., 2012. Systemic inflammation induces acute working memory deficits in the primed brain: relevance for delirium. Neurobiol Aging 33, 603-616 e603.

Murray, C.L., Skelly, D.T., Cunningham, C., 2011. Exacerbation of CNS inflammation and neurodegeneration by systemic LPS treatment is independent of 
circulating IL-1beta and IL-6. J Neuroinflammation 8, 50.

Osburg, B., Peiser, C., Domling, D., Schomburg, L., Ko, Y.T., Voigt, K., Bickel, U., 2002. Effect of endotoxin on expression of TNF receptors and transport of TNF-alpha at the blood-brain barrier of the rat. Am J Physiol Endocrinol Metab 283, E899-908.

Osorio, F., Reis e Sousa, C., 2011. Myeloid C-type lectin receptors in pathogen recognition and host defense. Immunity 34, 651-664.

Palin, K., McCusker, R.H., Strle, K., Moos, F., Dantzer, R., Kelley, K.W., 2008. Tumor necrosis factor-alpha-induced sickness behavior is impaired by central administration of an inhibitor of c-jun N-terminal kinase. Psychopharmacology (Berl) 197, 629-635.

Pecchi, E., Dallaporta, M., Thirion, S., Salvat, C., Berenbaum, F., Jean, A., Troadec, J.D., 2006. Involvement of central microsomal prostaglandin E synthase-1 in IL-1beta-induced anorexia. Physiol Genomics 25, 485-492.

Perry, V.H., 2010. Contribution of systemic inflammation to chronic neurodegeneration. Acta Neuropathol 120, 277-286.

Quan, N., Whiteside, M., Herkenham, M., 1998. Time course and localization patterns of interleukin-1beta messenger RNA expression in brain and pituitary after peripheral administration of lipopolysaccharide. Neuroscience 83, 281-293.

Renault, P.F., Hoofnagle, J.H., Park, Y., Mullen, K.D., Peters, M., Jones, D.B., Rustgi, 
V., Jones, E.A., 1987. Psychiatric complications of long-term interferon alfa therapy. Arch Intern Med 147, 1577-1580.

Romeo, H.E., Tio, D.L., Rahman, S.U., Chiappelli, F., Taylor, A.N., 2001. The glossopharyngeal nerve as a novel pathway in immune-to-brain communication: relevance to neuroimmune surveillance of the oral cavity. J Neuroimmunol 115, 91-100.

Rosin, D.L., Okusa, M.D., 2011. Dangers within: DAMP responses to damage and cell death in kidney disease. J Am Soc Nephrol 22, 416-425.

Saleh, M., 2011. The machinery of Nod-like receptors: refining the paths to immunity and cell death. Immunol Rev 243, 235-246.

Schiltz, J.C., Sawchenko, P.E., 2002. Distinct brain vascular cell types manifest inducible cyclooxygenase expression as a function of the strength and nature of immune insults. J Neurosci 22, 5606-5618.

Sharma, H.S., Hoopes, P.J., 2003. Hyperthermia induced pathophysiology of the central nervous system. Int J Hyperthermia 19, 325-354.

Singh, A.K., Jiang, Y., 2004. How does peripheral lipopolysaccharide induce gene expression in the brain of rats? Toxicology 201, 197-207.

Stefferl, A., Hopkins, S.J., Rothwell, N.J., Luheshi, G.N., 1996. The role of TNF-alpha in fever: opposing actions of human and murine TNF-alpha and interactions with 
IL-beta in the rat. Br J Pharmacol 118, 1919-1924.

Suehiro, E., Fujisawa, H., Ito, H., Ishikawa, T., Maekawa, T., 1999. Brain temperature modifies glutamate neurotoxicity in vivo. J Neurotrauma 16, 285-297.

Swain, M.G., Le, T., 1998. Chronic cholestasis in rats induces anhedonia and a loss of social interest. Hepatology 28, 6-10.

Teeling, J.L., Cunningham, C., Newman, T.A., Perry, V.H., 2010. The effect of non-steroidal anti-inflammatory agents on behavioural changes and cytokine production following systemic inflammation: Implications for a role of COX-1. Brain Behav Immun 24, 409-419.

Teeling, J.L., Felton, L.M., Deacon, R.M., Cunningham, C., Rawlins, J.N., Perry, V.H., 2007. Sub-pyrogenic systemic inflammation impacts on brain and behavior, independent of cytokines. Brain Behav Immun 21, 836-850.

Thornton, P., McColl, B.W., Cooper, L., Rothwell, N.J., Allan, S.M., 2010. Interleukin-1 drives cerebrovascular inflammation via MAP kinase-independent pathways. Curr Neurovasc Res 7, 330-340.

van Dam, A.M., Brouns, M., Louisse, S., Berkenbosch, F., 1992. Appearance of interleukin-1 in macrophages and in ramified microglia in the brain of endotoxin-treated rats: a pathway for the induction of non-specific symptoms of sickness? Brain Res 588, 291-296. 
van Munster, B.C., Bisschop, P.H., Zwinderman, A.H., Korevaar, J.C., Endert, E., Wiersinga, W.J., van Oosten, H.E., Goslings, J.C., de Rooij, S.E., 2010. Cortisol, interleukins and S100B in delirium in the elderly. Brain Cogn 74, 18-23.

van Munster, B.C., Korevaar, J.C., Zwinderman, A.H., Levi, M., Wiersinga, W.J., De Rooij, S.E., 2008. Time-course of cytokines during delirium in elderly patients with hip fractures. J Am Geriatr Soc 56, 1704-1709.

Vitkovic, L., Konsman, J.P., Bockaert, J., Dantzer, R., Homburger, V., Jacque, C., 2000. Cytokine signals propagate through the brain. Mol Psychiatry 5, 604-615.

Wan, W., Wetmore, L., Sorensen, C.M., Greenberg, A.H., Nance, D.M., 1994. Neural and biochemical mediators of endotoxin and stress-induced c-fos expression in the rat brain. Brain Res Bull 34, 7-14. 


\section{Figure Captions}

Figure 1. Summary diagram illustrating the relationships between systemic inflammation, cytokines, sickness, and delirium. Starting from the top, systemic infection and tissue injury respectively give rise to pathogen-associated molecular patterns (PAMPs) and alarmins, which both act on innate immune cells to trigger production of cytokines such as IL-1 $\beta$, IL-6, TNF- $\alpha$. These cytokines not only orchestrate local and distant inflammatory changes, but can also communicate with the brain via the fast neural and the slow humoural routes to induce sickness. It is believed that activation of the fast neural routes sensitizes the brain to the effects of the slow humoural routes, thereby amplifying sickness responses. Alternatively, infections and/or injury can generate non-cytokine mediators of sickness, including circulating prostaglandin E2 and LPS, which can both directly take effects in the brain and cause sickness. Although sickness is an adaptive response to facilitate recovery, in extreme cases it may manifest as delirium if uncontrolled. In young and healthy individuals, elevated cytokine levels by systemic inflammatory events lead to sickness. However, in the presence of other risk factors such as having an old age and/or dementia (e.g. Alzheimer's disease, AD), the same cytokines increases could possibly precipitate delirium. 
Tables

\begin{tabular}{|c|c|c|c|}
\hline & $\operatorname{PRR}(s)$ & PAMP & Pathogen \\
\hline \multirow[t]{9}{*}{ TLRs } & TLR1-TLR2 & Diacyl-lipopeptides & Bacteria \\
\hline & TLR2-TLR6 & Zymosan & Fungi \\
\hline & TLR2 & Peptidoglycan & Bacteria \\
\hline & TLR3 & dsRNA & Viruses \\
\hline & TLR4 & Lipopolysaccharide & Bacteria \\
\hline & TLR5 & Flagellin & Bacteria \\
\hline & TLR7 & ssRNA & Viruses \\
\hline & TLR8 & ssRNA & Viruses \\
\hline & TLR9 & Unmethylated CpG DNA & Bacteria \\
\hline \multirow[t]{4}{*}{ NLRs } & NOD1 & g-D-glutamyl-meso-diaminopimelic acid & Bacteria \\
\hline & NOD2 & Muramyl dipeptide & Bacteria \\
\hline & NALP1 & Anthrax toxin & Bacteria \\
\hline & NALP3 & Muramyl dipeptide & Bacteria \\
\hline \multirow[t]{2}{*}{ RLRs } & RIG-I & 5'-PPP ssRNA, short ssRNA & Viruses \\
\hline & MDAP5 & dsRNA & Viruses \\
\hline \multirow[t]{2}{*}{ CLRs } & Dectin-I & $\beta$-Glucan & Fungus \\
\hline & Mannose receptor & Mannose-capped lipoarabinomannan & Bacteria \\
\hline
\end{tabular}

Table 1. Pathogen recognition receptors (PRRs), their respective pathogen-associated molecular patterns (PAMPs) and pathogens, are summarized. Note that TLR1 and TLR2 form heterodimers with TLR2 and TLR6 respectively. Abbreviations: CLRs, C-type lectin receptors; MDA5, melanoma-differentiation-associated gene 5; NALP1 \& 3, NACHT, LRR and PYD domains-containing protein $1 \& 3$; NLRs, NOD-like receptors; NOD1 \& 2, nucleotide-binding oligomerization domain-containing protein $1 \&$ 2; RIG-I, retinoic-acid-inducible protein I; RLRs, RIG-I-like receptors; TLRs, toll-like receptors 


\begin{tabular}{|l|l|}
\hline Alarmin & Putative receptor(s) \\
\hline High mobility group box 1 & TLR2, TLR4, TLR9 \\
S100 proteins & TLR4 \\
Heat shock proteins & TLR2, TLR4 \\
Uric acid & TLR2, TLR4, NALP3 \\
$\beta$-amyloid & NALP3 \\
Cathelicidins & TLR7, TLR9 \\
Defensins & TLR4 \\
IL-1 $\alpha$ & IL-1R \\
IL-33 & IL-1R \\
\hline
\end{tabular}

Table 2. Examples of alarmins and their putative receptors. Abbreviations: HMGB1, High mobility group box 1; NALP3, NACHT, LRR and PYD domains-containing protein 3; TLR, toll-like receptor 
Systemic infection<smiles>C[Te]</smiles>

PAMPs (e.g. LPS, dsRNA)

Tissue injury

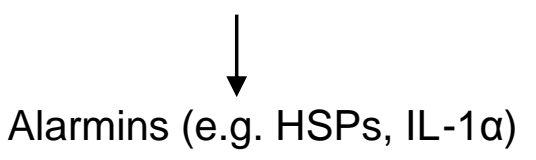

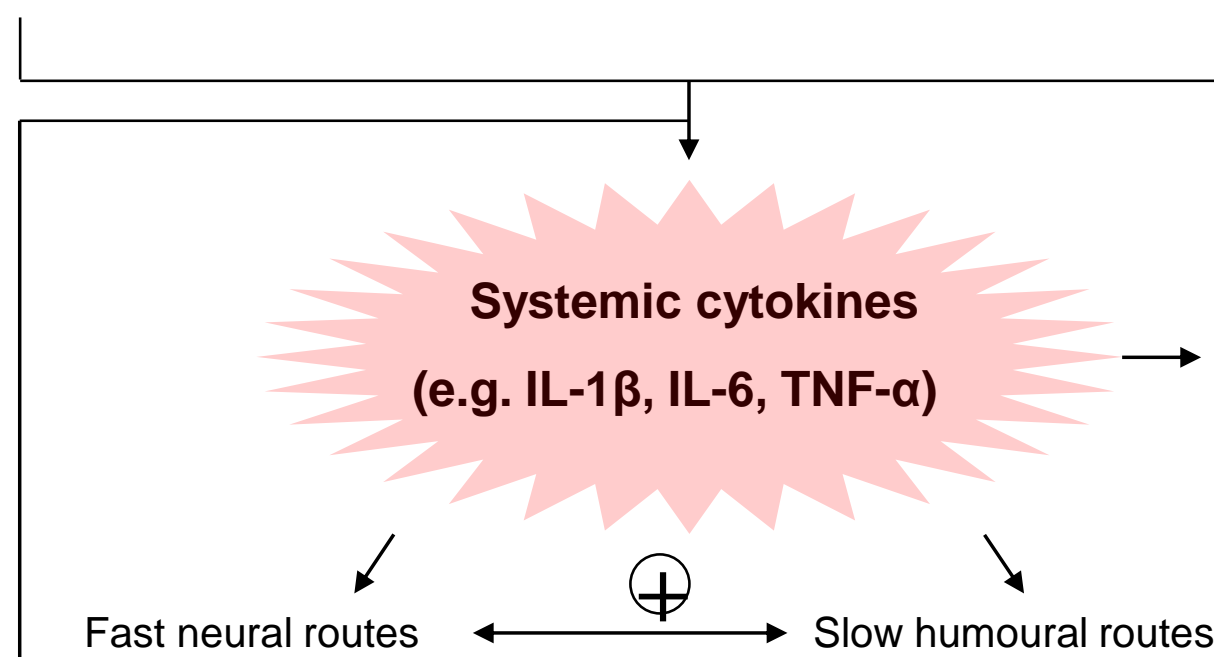

Systemic inflammation:

-Vasodilation

- Leukocyte infiltration

-Acute phase response

Other risk factors?

-Aging

-Dementia (e.g. AD)

\section{-LPS}

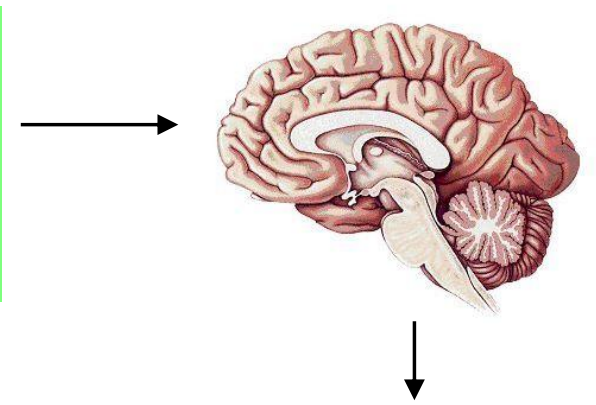

Sickness:

-Fever

-Annorexia

-Anhedonia
Delirium:

-Inattention

-Hallucinations

-Disorientation 